\title{
GaSb/GaAs quantum dot formation and demolition studied with cross-sectional scanning tunneling microscopy
}

\author{
E. P. Smakman, ${ }^{1, a)}$ J. K. Garleff, ${ }^{1}$ R. J. Young, ${ }^{2}$ M. Hayne, ${ }^{2}$ P. Rambabu, ${ }^{1}$ \\ and P. M. Koenraad ${ }^{1}$ \\ ${ }^{1}$ Department of Applied Physics, Eindhoven University of Technology, Eindhoven 5612 AZ, The Netherlands \\ ${ }^{2}$ Department of Physics, Lancaster University, Lancaster LAI 4YB, United Kingdom
}

(Received 20 December 2011; accepted 22 March 2012; published online 6 April 2012)

\begin{abstract}
We present a cross-sectional scanning tunneling microscopy study of $\mathrm{GaSb} / \mathrm{GaAs}$ quantum dots grown by molecular beam epitaxy. Various nanostructures are observed as a function of the growth parameters. During growth, relaxation of the high local strain fields of the nanostructures plays an important role in their formation. Pyramidal dots with a high Sb content are often accompanied by threading dislocations above them. GaSb ring formation is favored by the use of a thin GaAs first cap layer and a high growth temperature of the second cap layer. At these capping conditions, strain-driven $\mathrm{Sb}$ diffusion combined with $\mathrm{As} / \mathrm{Sb}$ exchange and $\mathrm{Sb}$ segregation remove the center of a nanostructure, creating a ring. Clusters of GaSb without a well defined morphology also appear regularly, often with a highly inhomogeneous structure which is sometimes divided up in fragments. @ 2012 American Institute of Physics. [http://dx.doi.org/10.1063/1.3701614]
\end{abstract}

Self-assembled quantum dots (QDs) are intensively studied for their unique opto-electronic properties. GaSb/ GaAs QDs have a type-II, staggered band alignment, in which holes are confined and electrons repelled from the QD region, resulting in a low recombination rate. Fast carrier capture and long charge storage capabilities of type-II QDs suggest their potential application in memory devices. ${ }^{1,2}$ Recent studies have shown that the use of a two-dimensional electron $^{3,4}$ or hole ${ }^{5,6}$ gas in combination with QDs allows writing and reading information, demonstrating the basic operations required in memory devices.

In the current work, we present an atomically resolved cross-sectional scanning tunneling microscopy (X-STM) study of $\mathrm{GaSb} / \mathrm{GaAs}$ QDs grown by molecular beam epitaxy (MBE). Previous X-STM studies have shown the structural properties of these QDs grown by metal-organic chemical vapor deposition ${ }^{7}$ and droplet epitaxy, ${ }^{8}$ the principles behind ring formation, ${ }^{9}$ and the spectroscopic features of these QDs. ${ }^{10}$ However, the dependence of the QD morphology on the growth parameters was never investigated. For memory applications, which generally require long storage times, the QDs should have a large hole localization energy. This property depends strongly on the shape and composition of a $\mathrm{QD},{ }^{11}$ thereby making detailed knowledge of the morphology important for future applications of these QDs.

The samples studied here consist of multiple layers of GaSb nanostructures embedded in GaAs, grown by MBE on an [001] oriented substrate, with a QD density $\sim 5 \times 10^{10} \mathrm{~cm}^{-2}$. The QD layers were grown on a GaAs buffer layer, after which 2 monolayers of $\mathrm{GaSb}$ were deposited, followed by a thin (4-16 nm) GaAs first cap layer. After a growth interrupt of 4 min under As flux, the GaAs second cap layer was grown at a temperature $\sim 100^{\circ} \mathrm{C}$ higher than the first cap layer. The overall growth temperatures ranged from 370 to $580^{\circ} \mathrm{C}$ and the III:V ratio during growth was kept constant at 1:5. Various samples were investigated to

${ }^{\text {a)} E l e c t r o n i c ~ m a i l: ~ e . p . s m a k m a n @ t u e . n l . ~}$ study the influence of the GaAs first cap thickness and the GaAs first and second cap temperatures on the morphology of the QDs.

All X-STM measurements were performed in an Omicron STM1 with a TS-2 scanner, under ultra-high vacuum conditions (pressure $\leq 6 \times 10^{-11} \mathrm{mbar}$ ) at room temperature. Electrochemically etched tungsten tips were used. The STM was operated in constant current mode to scan in situ cleaved cross-sectional $\{110\}$ surfaces. Applied bias voltages ranged between -2.5 and $-4.0 \mathrm{~V}$ with tunneling currents between 25 and $50 \mathrm{pA}$.

The QDs were grown epitaxially in Stranski-Krastanov mode. For GaSb on GaAs, the lattice mismatch is $8 \%$ and the strain energy of an $\mathrm{Ga}-\mathrm{Sb}$ pair $1.87 \mathrm{eV}$, which is larger compared to standard InAs/GaAs QDs where these are 7\% and $1.40 \mathrm{eV}$ for an In-As pair. ${ }^{9,12}$ In the active region of this material, various nanostructures are created during the growth process, see Figs. 1(a)-1(c). We observe three types of nanostructures: dots, rings, and clusters. Dots have the approximate shape of a truncated pyramid, with a nearly pure $\mathrm{Sb}$ content. The intermixing between $\mathrm{As}$ and $\mathrm{Sb}$ is substantially less than that was found between In and As in InAs/ GaAs QDs. ${ }^{13}$ Rings show up as two lobes of a cleaved torus, with a hole in the center that is almost entirely free of Sb, which is different from InAs/GaAs rings where In material is found inside the ring. ${ }^{12}$ Clusters have a strongly inhomogeneous $\mathrm{Sb}$ concentration, with widely varying morphologies. For some clusters, pure fragments of GaSb are observed with GaAs areas in between. The large lattice mismatch, high pair energy, and little intermixing of $\mathrm{Sb}$ and As lead to strong local strain fields around the nanostructures that are relaxed in different ways leading to varying morphologies.

In Fig. 2(a) a dot is shown with a screw dislocation nearby, which is shown in more detail in Fig. 2(b). It starts at the top of the dot and is terminated about $15 \mathrm{~nm}$ above it. Such structural deformations occur often around dot-shaped nanostructures with a nearly pure $\mathrm{Sb}$ content, relaxing the local strain. 

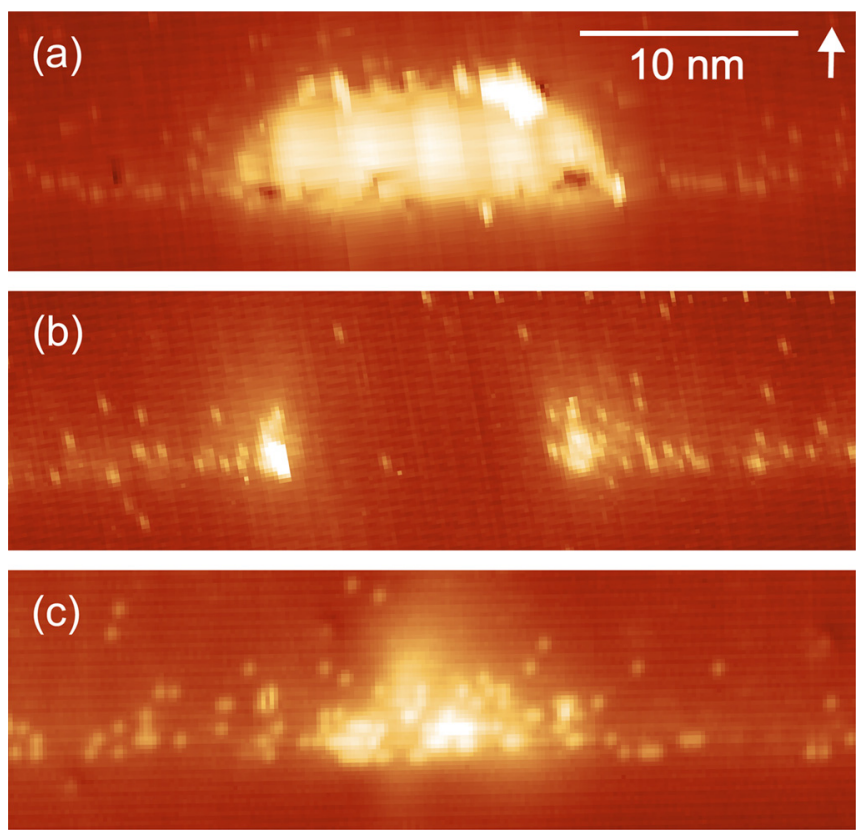

FIG. 1. Filled state X-STM images of various observed $\mathrm{GaSb} / \mathrm{GaAs}$ nanostructures. Typical examples of (a) a dot, (b) a centrally cleaved ring, and (c) a cluster. The growth direction is indicated by the arrow.

Free-standing GaSb QDs can also reduce the strain by evolving into rings when they are capped with GaAs. During the capping process, the local strain is initially enhanced because the QDs become encapsulated by material with a smaller lattice constant. ${ }^{14}$ The build-up of strain can then be relaxed by mass transport: $\mathrm{Sb}$ is removed from the dot by processes involving lateral diffusion, $\mathrm{As} / \mathrm{Sb}$ exchange, and Sb segregation. ${ }^{9}$ Diffusion of $\mathrm{Sb}$ occurs at regions with the highest local strain, such as the center of a QD. The instability arises at the position where the equilibrium of the interfacial surface tensions between the substrate, the QD, and the
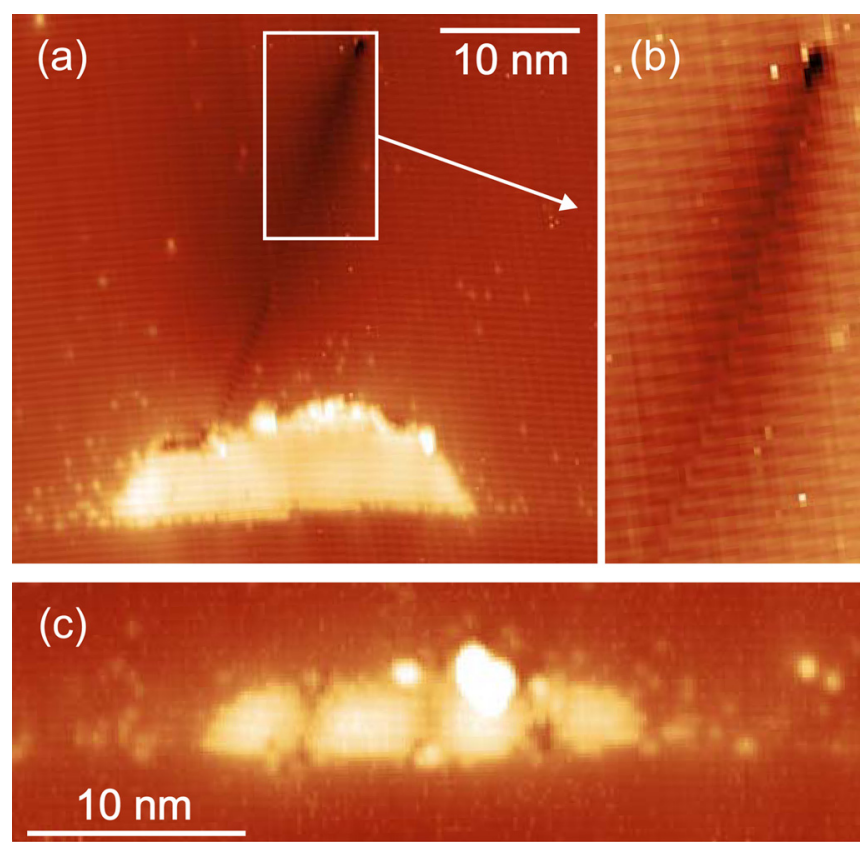

FIG. 2. Filled state X-STM images of (a) a dot with a screw dislocation on top, (b) a magnified portion of the dislocation revealing the termination at the top and the phase mismatch of the atomic rows at the dislocation boundary, and (c) a cluster with multiple segments of GaSb. cap layer are disturbed. ${ }^{14} \mathrm{As} / \mathrm{Sb}$ exchange and $\mathrm{Sb}$ diffusion empty those regions of $\mathrm{Sb}$, leading to rings with very little intermixing. This in contrast to what is found for InAs/GaAs rings, where strain driven diffusion is the main formation process and rings are not completely emptied of In. ${ }^{12}$

We argue that the observed clusters can form under similar conditions as the rings, but the exchange and segregation processes are incomplete (see, for example, Fig. 1(c)). Above these structures, we typically observe $\mathrm{Sb}$ rich regions. Some clusters contain multiple unstable regions from which the $\mathrm{Sb}$ is removed (see, for example, Fig. 2(c)), in which segments of $\mathrm{GaSb}$ are clearly visible with GaAs holes in between. They could be formed under the influence of interfacial misfit dislocations, which occur already at the beginning of the GaSb growth layer where they reduce the strain. ${ }^{15}$ The distance between the gaps in these clusters is 5-6 nm, which matches that of misfit dislocations measured on $\mathrm{GaSb} / \mathrm{GaAs}$ interfaces. ${ }^{16}$ However, with STM we were unable to observe any clear lattice defects around the base of these clusters. The limited occurrence of these structures prevented a more detailed study of possible misfit dislocations.

A statistical analysis was performed on four different samples that were grown with a range of growth parameters. In total, 374 nanostructures were analyzed with X-STM and classified. We found that the thickness of the first GaAs cap layer grown directly over the uncapped nanostructures strongly influences the outcome of the growth process, see Fig. 3(a), where the occurrence of various nanostructures is depicted as a function of the thickness of the first cap layer. For sufficiently thick cap layers, i.e., 8 and $16 \mathrm{~nm}$, a dot

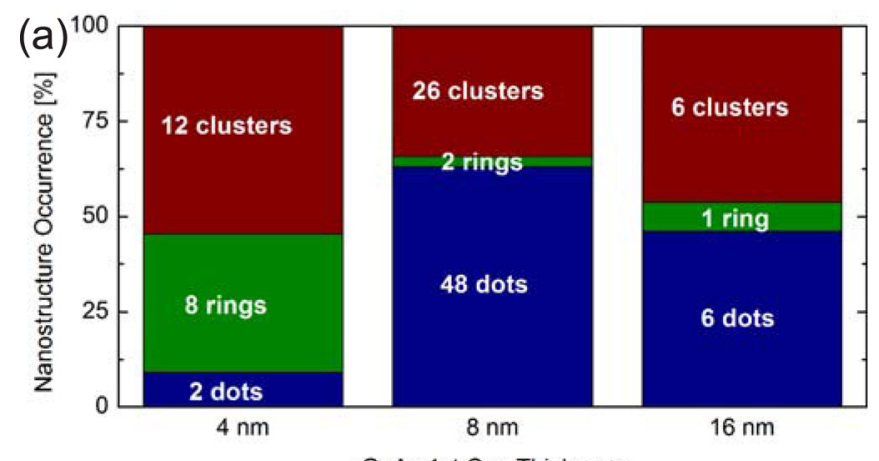

GaAs 1st Cap Thickness

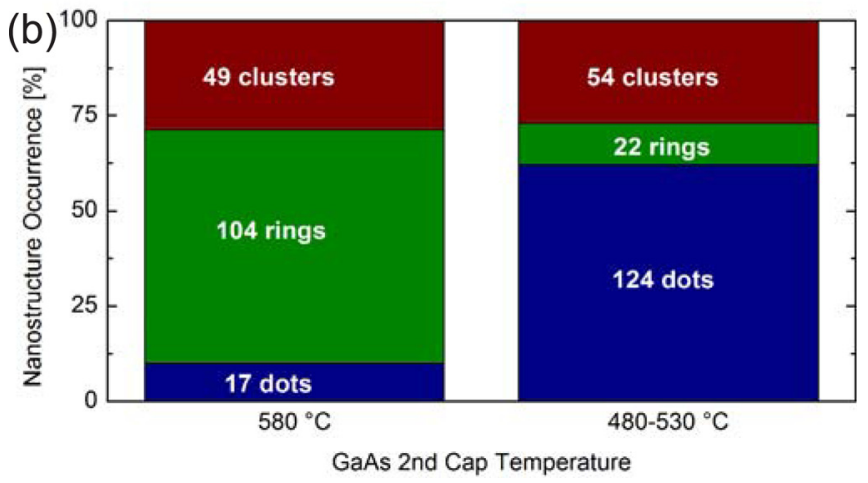

FIG. 3. Relative occurrence of dots (blue), rings (green), and clusters (red) grown with a range of growth conditions. The observed amounts of each type are indicated in the figure. (a) Various GaAs first cap thicknesses, where first and second cap temperatures always were 430 and $530{ }^{\circ} \mathrm{C}$, respectively. (b) Various GaAs second cap temperatures, where the first cap thickness always was $\geq 8 \mathrm{~nm}$. 


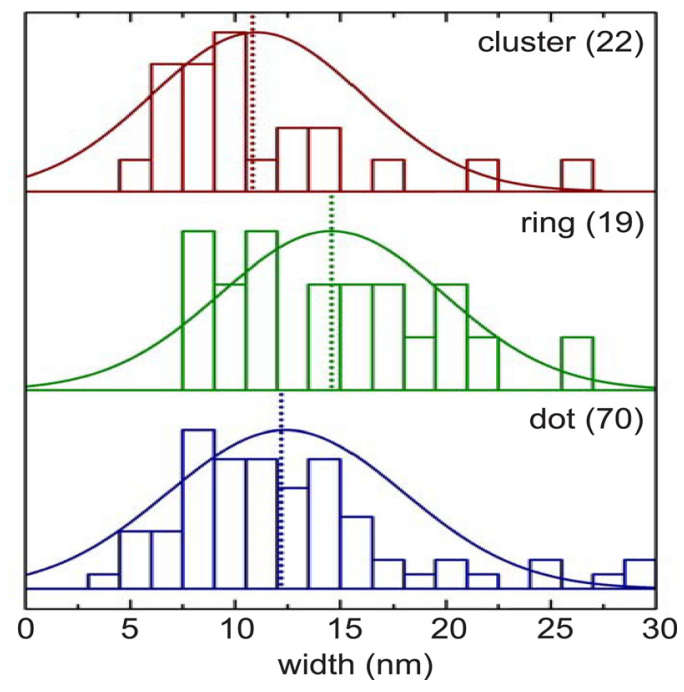

FIG. 4. Nanostructure width distributions (histograms) and Gaussian fits (lines) of dots (blue), rings (green), and clusters (red). Dashed lines indicate the middle of the distributions. Rings are on average about $20 \%$ wider than dots and clusters.

shape is preserved for $63 \%$ and $46 \%$ of the measured cases, respectively. For the $4 \mathrm{~nm}$ cap layer, the formation of rings is favored and only $9 \%$ of the dots survive. In this case, the uncapped QDs are not completely overgrown with the first cap layer, exposing them to the higher growth temperature of the second cap layer. The higher energy that is available for mass transport driven by diffusion, As/Sb exchange, and $\mathrm{Sb}$ segregation lead to more rings and fewer dots. The occurrence of clusters is not influenced significantly by variation of the growth conditions; they are always present in a concentration of about $25 \%-50 \%$.

The growth temperatures of the first and second cap layers were both varied, but only the second cap temperature showed a significant effect, which is shown in Fig. 3(b). In this case, all the QD layers were grown with a first cap thickness of $8 \mathrm{~nm}$ or higher, excluding the influence of partially uncapped QDs. For second cap temperatures in the range of $480-530^{\circ} \mathrm{C}$, dots are the predominant shape. For a second cap temperature of $580^{\circ} \mathrm{C}$, rings are favored. This supports the view that rings form during the capping process, when there is enough energy is available to enable mass transport. These results confirm previous studies performed by atomic force microscopy, where measurements on uncapped $\mathrm{GaSb}$ / GaAs nanostructures show only dots with no indication of holes in the center or other forms of dot demolition. ${ }^{11,17}$

For 112 nanostructures grown under similar growth conditions, the base width has been determined (see Fig. 4). Because the X-STM measurements are restricted to the cleavage plane, different cross-sections of the dots, rings, and clusters are imaged. This means that the width distributions are convolutions of the actual size of the nanostructures and the position of cleavage. However, the maxima of the fitted Gaussian distributions are a reasonable measure for com- paring the sizes of the different nanostructures. Clusters and dots are on average $20 \%$ smaller in width than rings. We suggest that ring formation is mostly favored in the presence of a large strain field. Smaller uncapped QDs remain dots often decorated with threading dislocations or evolve into clusters during the capping process. Some clusters could be formed around interfacial misfit dislocations that are already present before the capping process.

In conclusion, $\mathrm{GaSb} / \mathrm{GaAs}$ QDs give rise to a larger local strain as compared to standard InAs/GaAs QDs. This is due to larger lattice mismatch, higher pair energy, and less intermixing. The high local strain can be relaxed by means of threading dislocations above the dots, ring, or cluster formation by means of $\mathrm{Sb}$ segregation or fractioning of the nanostructures into clusters, possibly with misfit dislocations at their base. The occurrence of rings and dots strongly depend on the growth conditions, where the application of a thin first cap layer and high temperature growth of second cap layer favor the formation of rings over dots.

The funding for this work was provided by FOM (Grant 09NSE06) and EPSRC (Grant EP/H006419) in the framework of the NanoSci-E+ QD2D project and the Royal Society Brian Mercer Feasibility Award.

\footnotetext{
${ }^{1}$ A. Marent, M. Geller, and D. Bimberg, Microelectr. J. 40, 492 (2009).

${ }^{2}$ A. Marent, T. Nowozin, M. Geller, and D. Bimberg, Semicond. Sci. Tech. 26, 014026 (2011).

${ }^{3}$ B. Marquardt, M. Geller, A. Lorke, D. Reuter, and A. D. Wieck, Appl. Phys. Lett. 95, 022113 (2009).

${ }^{4}$ M. Geller, B. Marquardt, A. Lorke, D. Reuter, and A. D. Wieck, Nanoscale Res. Lett. 5, 829 (2010).

${ }^{5}$ A. Marent, T. Nowozin, J. Gelze, F. Luckert, and D. Bimberg, Appl. Phys. Lett. 95, 242114 (2009).

${ }^{6}$ T. Nowozin, A. Marent, G. Hönig, A. Schliwa, D. Bimberg, A. Beckel, B. Marquardt, A. Lorke, and M. Geller, Phys. Rev. B 84, 075309 (2011).

${ }^{7}$ R. Timm, H. Eisele, A. Lenz, S. K. Becker, J. Grabowski, T.-Y. Kim, L. Müller-Kirsch, K. Pötschke, U. W. Pohl, D. Bimberg, and M. Dähne, Appl. Phys. Lett. 85, 5890 (2004).

${ }^{8}$ B. Liang, A. Lin, N. Pavarelli, C. Reyner, J. Tatebayashi, K. Nunna, J. He, T. J. Ochalski, G. Huyet, and D. L. Huffaker, Nanotechnology 20, 455604 (2009).

${ }^{9}$ R. Timm, H. Eisele, A. Lenz, L. Ivanova, G. Balakrishnan, D. L. Huffaker, and M. Dähne, Phys. Rev. Lett. 101, 256101 (2008).

${ }^{10}$ R. Timm, H. Eisele, A. Lenz, L. Ivanova, V. Vossebürger, T. Warming, D. Bimberg, I. Farrer, D. A. Ritchie, and M. Dähne, Nano Lett. 10, 3972 (2010).

${ }^{11}$ M. Ahmad Kamarudin, M. Hayne, R. J. Young, Q. D. Zhuang, T. Ben, and S. Molina, Phys. Rev. B 83, 1 (2011).

${ }^{12}$ P. Offermans, P. M. Koenraad, J. H. Wolter, D. Granados, J. M. García, V. M. Fomin, V. N. Gladilin, and J. T. Devreese, Appl. Phys. Lett. 87, 131902 (2005).

${ }^{13}$ Q. Gong, P. Offermans, R. Nötzel, P. M. Koenraad, and J. H. Wolter, Appl. Phys. Lett. 85, 5697 (2004).

${ }^{14}$ R. Blossey and A. Lorke, Phys. Rev. E 65, 021603 (2002).

${ }^{15}$ G. Balakrishnan, J. Tatebayashi, A. Khoshakhlagh, S. H. Huang, A. Jallipalli, L. R. Dawson, and D. L. Huffaker, Appl. Phys. Lett. 89, 161104 (2006).

${ }^{16}$ S. H. Huang, G. Balakrishnan, A. Khoshakhlagh, A. Jallipalli, L. R. Dawson, and D. L. Huffaker, Appl. Phys. Lett. 88, 131911 (2006).

${ }^{17}$ M. Ahmad Kamarudin, M. Hayne, Q. D. Zhuang, O. Kolosov, T. Nuytten, V. V. Moshchalkov, and F. Dinelli, J. Phys. D 43, 065402 (2010).
} 